\title{
Free group automorphisms with parabolic boundary orbits
}

\author{
ARNAUD HILION
}

For $N \geqslant 4$, we show that there exist automorphisms of the free group $F_{N}$ which have a parabolic orbit in $\partial F_{N}$. In fact, we exhibit a technology for producing infinitely many such examples.

20E05, 20E36, 37B25, 37E15; 20F65, 37B05

\section{Introduction}

An automorphism $\varphi$ of the free group $F_{N}$ of rank $N$ induces a homeomorphism $\partial \varphi$ of the (Gromov) boundary $\partial F_{N}$ of $F_{N}$. The dynamics of the map $\partial \varphi$ on $\partial F_{N}$ has been studied a lot; see Levitt and Lustig $[13 ; 14 ; 15 ; 16]$ and the author's thesis [10]. We give a survey of the known results relevant in our context in Section 3. In this paper, we focus on the following question:

Does there exist an automorphism $\varphi$ of $F_{N}$ such that there is a parabolic orbit for the homeomorphism $\partial \varphi$ ?

We say that an automorphism $\varphi$ has a parabolic orbit if there exists two points $X, Y \in \partial F_{N}, X \neq Y$, such that

$$
\lim _{k \rightarrow \pm \infty} \partial \varphi^{k}(Y)=X
$$

We note that this implies that $X$ is a fixed point of $\partial \varphi$. In such a situation, the point $X \in \partial F_{N}$ is called a parabolic fixed point for $\varphi$, and the set $\left\{\partial \varphi^{k}(Y) \mid k \in \mathbb{Z}\right\}$ is called a parabolic orbit for $\varphi$. We prove:

Theorem 1.1 For $N \geqslant 4$ there exists an infinite family $\left\{\varphi_{k} \mid k \in \mathbb{N}\right\}$ of automorphisms of $F_{N}$ which have a parabolic orbit, such that for any $k, k^{\prime}, p, p^{\prime} \in \mathbb{N}, \varphi_{k}^{p}$ and $\varphi_{k^{\prime}}^{p^{\prime}}$ are conjugate if and only if $k=k^{\prime}$ and $p=p^{\prime}$.

Discussions with some of the experts of the subject have led the author to feel that the existence of such parabolic orbits come somehow as a surprise. To put Theorem 1.1 in perspective, we would like to mention the following three facts.

First, given a compact set $K$ and a homeomorphism $f$ of $K$, one says that $f$ has North-South dynamics, if (i) $f$ has precisely two distinct fixed points $x^{+}$and $x^{-}$, 
(ii) $\lim _{k \rightarrow+\infty} f^{k}(y)=x^{+}$and $\lim _{k \rightarrow+\infty} f^{-k}(y)=x^{-}$for all $y \in K \backslash\left\{x^{-}, x^{+}\right\}$, and (iii) the limit of $f^{k}$ when $k$ tends to infinity is uniform on compact subsets of $K \backslash\left\{x^{-}\right\}$and the limit of $f^{-k}$ is uniform on compact subsets of $K \backslash\left\{x^{+}\right\}$. It is proved in Levitt and Lustig [13] that "most" automorphisms of $F_{N}$, in a precise sense we do not explain here, have North-South dynamics on $\partial F_{N}$. In particular, they cannot have a parabolic orbit.

Second, let $\delta$ be the automorphism of $F_{2}=\langle a, b\rangle$ defined by $\delta(a)=a$ and $\delta(b)=b a$. The outer automorphism class $D$ of $\delta$ is sometimes called a Dehn twist automorphism. The reader, who has in mind the action by isometries of $\mathrm{SL}_{2}(\mathbb{Z})$ on the hyperbolic plane, should be warned that Dehn twist automorphisms do not give rise to parabolic orbits in $\partial F_{2}$. We give in Section 6 a description of all possible dynamics of automorphisms of $F_{2}$ in the outer class $D^{n}$, for $n \in \mathbb{Z}$.

Third, more generally, it is known that geometric automorphisms of $F_{N}$ do not have parabolic orbits in $\partial F_{N}$. We recall that an automorphism $\varphi$ of $F_{N}$ is geometric if there exist a surface $S$ (with nonempty boundary) with fundamental group $\pi_{1}(S)$ isomorphic to $F_{N}$ and a homeomorphism $f$ of $S$ which induces $\varphi$ on $F_{N} \cong \pi_{1}(S)$. More details are given in Section 4.2. As a consequence, since all automorphisms of $F_{2}$ are known to be geometric, one obtains:

Proposition 1.2 No automorphism of $F_{2}$ has a parabolic orbit.

To our knowledge, the question of the existence of automorphisms with a parabolic orbit is still open for $F_{3}$.

Acknowledgments I would like to express my gratitude to Gilbert Levitt, who has posed the question of the existence of parabolic orbits as part of my thesis project [10], and has consistently encouraged me to publish my results since then. I would like to thank Pascal Hubert and Erwan Lanneau for helpful discussions about dilatation coefficients of matrices in $\mathrm{SL}_{2}(\mathbb{Z})$. I am grateful to Martin Lustig for his active interest in the present paper.

This work has been supported by the Agence Nationale de la Recherche grant number ANR-10-JCJC 01010.

\section{A first example}

For the impatient reader, we give a first example of an automorphism of $F_{4}=\langle a, b, c, d\rangle$ with a parabolic orbit "inside $F_{4}$ " (using Proposition 3.5, this gives immediately a parabolic orbit in $\left.\partial F_{4}\right)$. 
Let $\varphi$ be the automorphism defined by

$$
\begin{aligned}
\varphi: & \mapsto \mapsto a \\
b & \mapsto b a \\
c & \mapsto c a^{2} \\
d & \mapsto d c .
\end{aligned}
$$

The inverse of $\varphi$ is given by

$$
\begin{aligned}
\varphi^{-1}: a & \mapsto a \\
b & \mapsto b a^{-1} \\
c & \mapsto c a^{-2} \\
d & \mapsto d a^{2} c^{-1} .
\end{aligned}
$$

The common limit point of the forward and backward iteration of $\varphi$ (called a "parabolic fixed point") will be the element $b a^{-\infty}=b a^{-1} a^{-1} a^{-1} a^{-1} \cdots \in \partial F_{4}$. The element of $F_{4}$ which gives rise to a parabolic orbit with this limit point is $b d^{-1}$. We calculate:

$$
\begin{aligned}
& b d^{-1} \stackrel{\varphi}{\mapsto} b a c^{-1} \cdot d^{-1} \stackrel{\varphi}{\mapsto} b c^{-1} \cdot c^{-1} d^{-1} \stackrel{\stackrel{\varphi}{\mapsto}}{ } b a^{-1} c^{-1} \cdot a^{-2} c^{-1} c^{-1} d^{-1} \\
& \stackrel{\varphi}{\mapsto} b a^{-2} c^{-1} \cdot a^{-4} c^{-1} a^{-2} c^{-1} c^{-1} d^{-1} \stackrel{\varphi}{\mapsto} \cdots \\
& b \cdot d^{-1} \stackrel{\varphi^{-1}}{\mapsto} b a^{-1} \cdot c a^{-2} d^{-1} \stackrel{\varphi^{-1}}{\mapsto} b a^{-2} \cdot c a^{-4} c a^{-2} d^{-1} \\
& \stackrel{\varphi^{-1}}{\mapsto} b a^{-3} \cdot c a^{-6} c a^{-4} c a^{-2} d^{-1} \stackrel{\varphi^{-1}}{\mapsto} \cdots
\end{aligned}
$$

In these calculations, we help the reader to follow through the iteration by introducing an extra - which is "mapped" to the · in the next iteration step. The crucial feature is that at any of these $\cdot$ no cancellation does occur. We see that $\lim _{k \rightarrow+\infty} \varphi^{k}\left(b d^{-1}\right)=$ $\lim _{k \rightarrow+\infty} \varphi^{-k}\left(b d^{-1}\right)=b a^{-\infty}$. A more formal justification is given in Section 5 .

\section{Basics}

This section serves sort of as glossary: We summarize in a sequence of brief subsections the basic definitions and facts which are needed to follow the arguments in the subsequent sections. The expert reader is encouraged to skip the first few subsections (and to go back later to them, if need be). However, the terminology introduced in the last subsections is nonstandard and should be read carefully. 


\subsection{The induced boundary homeomorphism}

Let $F_{N}$ denote the free group of finite rank $N \geqslant 2$. The boundary $\partial F_{N}$ of $F_{N}$ is a Cantor set. If $\mathcal{A}=\left\{a_{1}, \ldots, a_{N}\right\}$ is a basis of $F_{N}$, we denote by $\mathcal{A}^{ \pm 1}$ the set $\left\{a_{1}, \ldots, a_{N}, a_{1}^{-1}, \ldots, a_{N}^{-1}\right\}$. A word $w=w_{1} \cdots w_{p}\left(w_{i} \in \mathcal{A}^{ \pm 1}\right)$ is reduced if $w_{i+1} \neq w_{i}^{-1}$. The free group $F_{N}$ can be understood as the set of (finite) reduced words in $\mathcal{A}^{ \pm 1}$. Then the boundary $\partial F_{N}$ is naturally identified to the set of (right) infinite reduced words $X=x_{1} \cdots x_{p} \ldots$ with $x_{i} \in \mathcal{A}^{ \pm 1}, x_{i+1} \neq x_{i}^{-1}$. The cylinder defined by a reduced word $w=w_{1} \cdots w_{p}$ is the set of right-infinite reduced words $X=x_{1} \cdots x_{k} \cdots$ which admit $w$ as prefix: $x_{i}=w_{i}$ for $i \in\{1, \ldots, p\}$. A basis of topology of $\partial F_{N}$ is given by the set of all such cylinders.

An automorphism $\varphi$ of a free group $F_{N}$ induces a homeomorphism $\partial \varphi$ of the boundary $\partial F_{N}$. This can easily be checked by considering a standard set of generators of the automorphisms group $\operatorname{Aut}\left(F_{N}\right)$ of $F_{N}$. Alternatively, this can be seen as a consequence of the fact that a quasi-isometry of a proper Gromov-hyperbolic space induces a homeomorphism on the boundary of this space; see Ghys and de la Harpe [7]. Indeed, $F_{N}$ equipped with the word metric associated to a basis $\mathcal{A}$, is a proper Gromov0 -hyperbolic space, and any automorphism of $F_{N}$ is a quasi-isometry of $F_{N}$ with respect to this metric.

\subsection{Compactification of $F_{N}$}

Let $\bar{F}_{N}$ denote the union of $F_{N}$ and its boundary $\partial F_{N}$, ie $\bar{F}_{N}=F_{N} \cup \partial F_{N}$. Given a basis of $F_{N}$, if $w$ is a reduced word, let $C_{w}$ be the set of reduced finite or infinite words which have $w$ as prefix. A basis of topology of $\bar{F}_{N}$ is given by the finite subsets of $F_{N}$ and the sets $C_{w}$ (with $w$ describing all the reduced words of $F_{N}$ ). Then $\bar{F}_{N}$ is a compact set, and the inclusions of $F_{N}$ and $\partial F_{N}$ in $\bar{F}_{N}$ are embeddings. If $\varphi$ is an automorphism of $F_{N}, \bar{\varphi}$ will denote the map defined by $\bar{\varphi}(g)=\varphi(g)$ if $g \in F_{N}$ and $\bar{\varphi}(X)=\partial \varphi(X)$ if $X \in \partial F_{N}$. The map $\bar{\varphi}$ is a homeomorphism of $\bar{F}_{N}$.

\subsection{Getting rid of periodicity}

Let $f$ be a homeomorphism of a topological space $\mathcal{X}$. We denote by $\operatorname{Fix}(f)=\{x \in \mathcal{X} \mid$ $f(x)=x\}$ the set of fixed points of $f$, and by $\operatorname{Per}(f)=\bigcup_{k \in \mathbb{N}} \operatorname{Fix}\left(f^{k}\right)$ the set of periodic points of $f$.

Levitt and Lustig have proved in [14] that there exists an integer $p$, which depends only on the rank $N$ of $F_{N}$, such that for all $\varphi \in \operatorname{Aut}\left(F_{N}\right)$, the periodic points of $\bar{\varphi}^{p}$ are fixed points: $\operatorname{Fix}\left(\bar{\varphi}^{p}\right)=\operatorname{Per}\left(\bar{\varphi}^{p}\right)$. This result has been refined by Feighn and 
Handel in [5], where the notion of "forward rotationless" outer automorphism has been introduced. This lead us to say, in this paper, that an automorphism $\varphi \in \operatorname{Aut}\left(F_{N}\right)$ is rotationless if $\operatorname{Fix}(\bar{\varphi})=\operatorname{Per}(\bar{\varphi})$. The previously mentioned result can be rephrased as follows:

Theorem 3.1 (Levitt-Lustig) Any automorphism $\varphi \in \operatorname{Aut}\left(F_{N}\right)$ has a power $\varphi^{p}$ $(p \in \mathbb{N})$ which is rotationless.

\subsection{Nature of fixed points}

Let $\varphi$ be a rotationless automorphism of $F_{N}$. The set $\operatorname{Fix}(\varphi)$ is a subgroup of $F_{N}$, which is called the fixed subgroup of $\varphi$. This fixed subgroup has finite rank; see Cooper [4]. More precisely, Bestvina and Handel [2] proved that $\operatorname{rank}(\operatorname{Fix}(\varphi)) \leqslant N$. In particular, $\operatorname{Fix}(\varphi)$ is a quasiconvex subgroup of $F_{N}$, and thus its boundary $\partial \operatorname{Fix}(\varphi)$ naturally injects into $\partial F_{N}$. By continuity of $\bar{\varphi}$, every point of $\partial \operatorname{Fix}(\varphi)$ is contained in Fix $(\partial \varphi)$. Following Nielsen, these fixed points of $\partial \varphi$ are called singular; the fixed points of $\partial \varphi$ which are not singular are called regular.

A fixed point $X$ of $\partial \varphi$ is attracting if there exists a neighbourhood $U$ of $X$ in $\bar{F}_{N}$ such that the sequence $\bar{\varphi}^{k}(x)$ converges to $X$ for all $x$ in $U$. A fixed point $X$ of $\partial \varphi$ is repulsing if it is attracting for $\partial \varphi^{-1}$. Gaboriau et al [6] proved that:

Lemma 3.2 Let $\varphi \in \operatorname{Aut}\left(F_{N}\right)$. A regular fixed point of $\partial \varphi$ is either attracting or repulsing.

However, outside of the regular fixed point set, ie for singular fixed points, the dynamics can be quite a bit more complicated. In particular, there may exist mixed fixed points, ie fixed points which serve as attractor for some orbits, and simultaneously as repeller for others. This phenomenon is rather common; some concrete examples will be spelled out in the subsequent sections.

A particular case of a mixed fixed point is the case (defined in the Introduction) of a parabolic fixed point. Thus we obtain as special case the following consequence of Lemma 3.2:

Remark 3.3 Any parabolic fixed point of $\varphi$ is singular.

\subsection{Limit points}

Let $\varphi$ be a rotationless automorphism of $F_{N}$. For any $x \in \bar{F}_{N}$, if $\lim _{k \rightarrow+\infty} \bar{\varphi}^{k}(x)$ exists, we denote it by $\omega_{\varphi}(x)$. In [16], Levitt and Lustig proved: 
Theorem 3.4 (Levitt-Lustig) Let $\varphi \in \operatorname{Aut}\left(F_{N}\right)$ be rotationless. Then for any $x \in \bar{F}_{N}$ the sequence $\bar{\varphi}^{k}(x)$ converges to some element $\omega_{\varphi}(x) \in \operatorname{Fix}(\bar{\varphi})$.

A point $X \in \partial \operatorname{Fix}(\varphi)$ is a $\omega$-limit point of $\varphi$ if there exists $x \in \bar{F}_{N}$ such that $X=\omega_{\varphi}(x)$. A point $X \in \partial \operatorname{Fix}(\varphi)$ is a limit point of $\varphi$ if it is a $\omega$-limit point of $\varphi$ or $\varphi^{-1}$. Let $L_{\varphi}^{\omega}$ denote the set of $\omega$-limit points of $\varphi$ and let $L_{\varphi}$ denote the set of limit points of $\varphi$.

For any $g \in F_{N}, g \neq 1$, the sequence $g^{k}$ has a limit in $\partial F_{N}$ when $k \rightarrow+\infty$ : this limit is denoted by $g^{\infty}$.

Proposition 3.5 Let $\varphi \in \operatorname{Aut}\left(F_{N}\right)$ be a rotationless automorphism. If $g \in F_{N} \backslash \operatorname{Fix}(\varphi)$, then

$$
\omega_{\varphi}(g)=\omega_{\varphi}\left(g^{\infty}\right)
$$

Proof The proof is a simple adaptation of the arguments in the proof of [13, Proposition 2.3]. We fix a basis $\mathcal{A}$ of $F_{N}$. We note that for all $g \in F_{N} \backslash\{1\}$, the Gromov product $\left(g, g^{\infty}\right)$ (ie the length of longest common prefix) of $g$ and $g^{\infty}$ is bigger than $\frac{1}{2}(|g|+1)$ (where $|g|$ denotes the length of $g$ in the basis $\mathcal{A}$ ). If $g \notin \operatorname{Fix}(\varphi)$, then the length of $\varphi^{k}(g)$, and thus also the Gromov product $\left(\varphi^{k}(g),\left(\varphi^{k}(g)\right)^{\infty}\right)$, tend to infinity. Theorem 3.4 implies that $\omega_{\varphi}(g)=\omega_{\varphi}\left(g^{\infty}\right)$.

Proposition 3.5 shows that $L_{\varphi}^{\omega}=\left\{\omega_{\varphi}(X) \mid X \in \partial F_{N}\right\}$. We do not know whether $L_{\varphi}^{\omega}=\left\{\omega_{\varphi}(g) \mid g \in F_{N}\right\}$ holds.

\subsection{Isoglossy classes}

For any $\varphi \in \operatorname{Aut}\left(F_{N}\right)$, two points $X, Y \in \partial F_{N}$ are called isogloss (with respect to $\varphi$ ) if there exists some $g \in \operatorname{Fix}(\varphi)$ such that $X=g Y$. It follows directly from this definition that isoglossy is an equivalence relation. The fixed subgroup Fix $(\varphi)$ acts naturally on the fixed point set Fix $(\partial \varphi)$, which is thus naturally partitioned into isoglossy classes. If $X, Y \in \operatorname{Fix}(\partial \varphi)$ are isogloss, then they are of same "dynamical type": they are simultaneously singular, attracting, repulsing, mixed, parabolic or limit points.

\subsection{Dynamics graph}

Let $\varphi \in \operatorname{Aut}\left(F_{N}\right)$ be a rotationless automorphism. We associate to $\varphi$ a graph $\Gamma_{\varphi}$, called the dynamics graph of $\varphi$. The vertices of $\Gamma_{\varphi}$ are the isoglossy classes of points of $L_{\varphi}$. There is an oriented edge from the isoglossy class $x_{1}$ to the isoglossy class $x_{2}$ if there exists some representatives $X_{i}$ of $x_{i}$ and $X \in \partial F_{N}$ such that $\omega_{\varphi^{-1}}(X)=X_{1}$ 
Figure 1. North-South dynamics graph

and $\omega_{\varphi}(X)=X_{2}$. The main theorem of [10] states that $\Gamma_{\varphi}$ is a finite graph. We give in Figure 1 the dynamics graph of an automorphism which has North-South dynamics on $\partial F_{N}$.

Finally, we note that, for a rotationless automorphism $\varphi$, the existence of parabolic orbit is equivalent to the fact that there is an edge of the dynamics graph $\Gamma_{\varphi}$ which is a loop.

Remark 3.6 In [11], Levitt introduces a graph in order to code the dynamics of socalled "simple-dynamics homeomorphisms" of the Cantor set $C$ : a homeomorphism $f: C \rightarrow C$ has simple dynamics if the set Fix $(f)$ of its fixed points is finite, and if the sequence $f^{n}$ uniformly converges on any compact set disjoint from $\operatorname{Fix}(f)$. If $\varphi \in \operatorname{Aut}\left(F_{N}\right)$ is a rotationless automorphism with trivial fixed subgroup, then $\partial \varphi$ has simple dynamics, and the graph $\Gamma_{\varphi}$ is the same as the one defined in [11]. In this case, the fixed points of $\partial \varphi$ are either attracting or repulsing. Thus, if one is interested in parabolic orbits, which are the main focus of the present paper, one has to purposefully leave to world of "simple dynamics" homeomorphisms.

\section{Examples}

\subsection{Inner automorphisms}

Let $i_{u} \in \operatorname{Aut}\left(F_{N}\right)$ denote the conjugation, or inner automorphism, by $u \in F_{N}$, ie $i_{u}(g)=u g u^{-1}$ for all $g \in F_{N}$. The set $\operatorname{Inn}\left(F_{N}\right)$ of inner automorphisms of $F_{N}$ is a normal subgroup of $\operatorname{Aut}\left(F_{N}\right)$. The quotient group, denoted by $\operatorname{Out}\left(F_{N}\right)$, is the group of outer automorphisms of $F_{N}$.

The homeomorphism $\partial i_{u}: \partial F_{N} \rightarrow \partial F_{N}$ induced by $i_{u}$ is the left translation by $u$ : $\partial i_{u}(X)=u X$. If $u \neq 1$, the map $\partial i_{u}$ has precisely 2 fixed points: $u^{\infty}$ and $u^{-\infty}$ (where $u^{\infty}$ is the limit of the sequence $u^{k}$, and $u^{-\infty}$ is the limit of the sequence $u^{-k}$, for $k \rightarrow+\infty)$. Moreover, for any point $X \in \partial F_{N}$ different from $u^{-\infty}$, the sequence $\partial \varphi^{k}(X)$ converges to $u^{\infty}$ when $k$ tends to infinity. One checks easily that the map $\partial i_{u}$ has North-South dynamics, from $u^{-\infty}$ to $u^{\infty}$, on $\partial F_{N}$; see [13] for instance. 
Remark 4.1 We note that the fixed subgroup of $i_{u}$ is cyclic, generated by the root of $u$ (ie the element $v \in F_{N}$ such that $u=v^{p}$ with $p \in \mathbb{N}$ maximal). In particular, $u^{\infty}$ and $u^{-\infty}$ are singular fixed points of $i_{u}$. This shows that when defining " $X$ is an attracting fixed point of $\varphi$ " in Section 3.4, it makes a crucial difference that we request the neighbourhood $U$ of $X$ to be taken in $\bar{F}_{N}$ and not just in $\partial F_{N}$.

\subsection{Geometric automorphisms}

Let $\Sigma$ be a compact surface with fundamental group $\pi_{1}(\Sigma)$ isomorphic to $F_{N}$ (in particular, $\Sigma$ has nonempty boundary). The surface $\Sigma$ can be equipped with a hyperbolic metric (ie a metric of constant curvature equal to -1 ) in such a way that every boundary component of the boundary of $\Sigma$ is a geodesic. The universal cover $\tilde{\Sigma}$ of $\Sigma$ is then identified with a closed convex subset of the hyperbolic plane $\mathbb{H}^{2}$, and the Gromov boundary $\partial \tilde{\Sigma}$ of $\tilde{\Sigma}$, which is naturally identified with the boundary $\partial F_{N}$ of $F_{N}$, injects in the boundary (or circle at infinity) $S_{\infty}$ of $\mathbb{H}^{2}$. Since $S_{\infty}$ is a circle, it can be equipped with a natural cyclic order. This order on $S_{\infty}$ induces a cyclic order on $\partial F_{N}$.

In his fundamental work $[19 ; 20 ; 21]$, Nielsen proposed an original and fruitful point of view to study homeomorphisms of surfaces. The basic idea is that the behaviour of a homeomorphism $f$ of a surface $\Sigma$ is well reflected by the collection of all the lifts $\tilde{f}$ of $f$ to $\widetilde{\Sigma}$ which have each much simpler individual behaviour. This idea is at the origin of what is now called "Nielsen-Thurston classification" of homeomorphisms of surfaces (see Handel and Thurston [9]), and it has much influenced the study of (outer) automorphisms of free groups (see Gaboriau et al [6], Feighn and Handel [5], Handel and Mosher [8]). The key fact is that any lift $\tilde{f}$ of $f$ induces a homeomorphism $\partial \tilde{f}$ of $\partial \tilde{\Sigma}$. A basic (but rather fundamental) remark is that $\partial \tilde{f}$ preserves the cyclic order on $\partial \widetilde{\Sigma} \subseteq S_{\infty}$.

An homeomorphism $f$ of $\Sigma$ induces an outer automorphism of $\pi_{1}(\Sigma)$, and thus an outer automorphism $\Phi \in \operatorname{Out}\left(F_{N}\right)$ (in fact, this outer automorphism $\Phi$ only depends on the mapping class of $f$ ). Such an outer automorphism $\Phi$ of $F_{N}$ (and also any automorphism $\varphi \in \Phi)$ is called geometric. Classical Galois theory for covering spaces states that the lifts of $f$ are in bijective correspondence with the automorphisms in the outer class $\Phi$. More precisely, an automorphism $\varphi \in \Phi$ and a lift $\tilde{f}$ of $f$ are in correspondence if and only if

$$
\varphi(g) \circ \tilde{f}=\tilde{f} \circ g \quad \forall g \in F_{N},
$$

where the elements of $F_{N}$ are considered as deck transformations of $\widetilde{\Sigma}$. As a consequence, the dynamics of $\partial \tilde{f}$ on $\partial \widetilde{\Sigma}$ and the dynamics of $\partial \varphi$ on $\partial F_{N}$ are conjugate via the natural identification between $\partial \widetilde{\Sigma}$ and $\partial F_{N}$. 
It follows from the previous discussion that, for any geometric automorphism $\varphi \in$ $\operatorname{Aut}\left(F_{N}\right)$, the homeomorphism $\partial \varphi$ of $\partial F_{N}$ must preserve a cyclic order on $\partial F_{N}$.

Another fact proved by Nielsen is that $\partial \tilde{f}$ has at least 2 periodic points on $\partial F_{N}$ (for a proof in the context of free groups; see [14]). This means that there exists a positive power of $\partial \tilde{f}$ which has at least 2 fixed points on $\partial F_{N}$. Both these facts (existence of 2 fixed points and preservation of a cyclic order) yield directly:

Proposition 4.2 A geometric automorphism of $F_{N}$ cannot have a parabolic orbit in $\partial F_{N}$.

This fact is particularly meaningful for the free group of rank 2 . Indeed, it is well known that any outer automorphism of $F_{2}$ can be induced by a homeomorphism of a torus with one boundary component; see Nielsen [18]. This is precisely how Proposition 1.2 is proved.

\subsection{Outer automorphisms}

Although well known, we believe that at this point it might be wise to alert the less expert reader about a common misunderstanding. It is by no means true that any two automorphisms $\varphi, \varphi^{\prime}$ which belong to the same outer automorphism class $\Phi$, must have conjugate dynamics. Indeed, their dynamics graphs $\Gamma_{\varphi}$ and $\Gamma_{\varphi^{\prime}}$ may look quite different. Concrete examples are easy to come by, and some are given in the subsequent sections.

The reader who wants to be more subtle can easily check that indeed some automorphisms in $\Phi$ have naturally conjugate dynamics. The resulting isogredience classes go again all the way back to Nielsen (see also [13]), and one could associate to $\Phi$ a total dynamics graph which is the disjoint union of the $\Gamma_{\varphi}$ over a set of representatives for the single isogredience classes. However, this goes beyond the scope of this paper.

\section{Parabolic orbits}

\subsection{Structure of a parabolic fixed point}

Let $\varphi \in \operatorname{Aut}\left(F_{N}\right)$ be an automorphism, and $X \in \operatorname{Fix}(\partial \varphi)$ be a parabolic fixed point for $\varphi$. We have seen (cf Remark 3.3) that $X$ must be singular. A point $X \in \partial F_{N}$ is rational if it a fixed point of an inner automorphism, ie $X=u^{\infty}$ for some $u \in F_{N} \backslash\{1\}$. It is proved in [10] that singular limit points of $\varphi$ are rational. We deduce the following: 
Lemma 5.1 A parabolic fixed point $X$ of $\varphi \in \operatorname{Aut}\left(F_{N}\right)$ is a singular rational point: $X=u^{\infty}$ with $u \in \operatorname{Fix}(\varphi)$.

Moreover, we have:

Proposition 5.2 Let $\varphi$ be an automorphism of $F_{N}$, and $X \in \operatorname{Fix}(\partial \varphi)$ be a parabolic fixed point for $\varphi$. Then any neighborhood of $X$ in $\partial F_{N}$ contains a full orbit $\left\{\partial \varphi^{k}(Y) \mid\right.$ $k \in \mathbb{Z}\} \subset \partial F_{N}$

Proof We have seen that $X=u^{\infty}$, with $u \in \operatorname{Fix}(\varphi)$. We consider a given neighborhood $\mathcal{V}$ of $X$. Let $\left.\vartheta=\left\{\partial \varphi^{k}(Y)\right) \mid k \in \mathbb{Z}\right\}$ be a parabolic orbit for $X$. We note that $\vartheta \cup\{X\}$ is a compact subset of $\partial F_{N}$. Moreover, $u^{-\infty} \notin \vartheta \cup\{X\}$ because $Y \notin \operatorname{Fix}(\partial \varphi)$. Since the sequence $\left(\partial i_{u}^{p}\right)_{p \in \mathbb{N}}$ uniformly converges on compact subsets of $\partial F_{N} \backslash\left\{u^{-\infty}\right\}$ towards $u^{\infty}$ when $p$ tends to infinity (see Section 4.1), the set $\partial i_{u}^{p}(\vartheta)$ is contained in $\mathcal{V}$, up to taking $p$ sufficiently large. We remark that, since $u \in \operatorname{Fix}(\varphi), \partial i_{u}^{p}\left(\partial \varphi^{k}(Y)\right)=\partial \varphi^{k}\left(u^{p} Y\right)$, and thus $\partial i_{u}^{p}(\vartheta)=u^{p} \vartheta$ is a parabolic orbit for $X$.

\subsection{Automorphisms of $F_{4}$ which have parabolic orbits}

For any $k \in \mathbb{N}$, consider the automorphism $\varphi_{k}$ of $F_{4}=\langle a, b, c, d\rangle$ given by

$$
\begin{aligned}
\varphi_{k}: a & \mapsto a \\
b & \mapsto b a \\
c & \mapsto c a^{k+1} \\
d & \mapsto d c
\end{aligned}
$$

and its inverse

$$
\begin{aligned}
\varphi_{k}^{-1}: & a \mapsto a \\
b & \mapsto b a^{-1} \\
c & \mapsto c a^{-k-1} \\
d & \mapsto d a^{k+1} c^{-1} .
\end{aligned}
$$

The rose $R_{4}$ is the geometric realization of graph with one vertex and 4 edges. We put an orientation on each edge, and we label them by $a, b, c$ and $d$. We can turn $R_{4}$ into a length space by declaring that each edge has length 1 . As usual, the automorphisms $\varphi_{k}^{ \pm 1}$ can be realized as homotopy equivalences $f_{k}^{ \pm}$of the rose $R_{4}$ where each edge is mapped linearly to the edge path with label preassigned by $\varphi_{k}^{ \pm 1}$.

In fact, the automorphisms $\varphi_{k}^{ \pm 1}$ define outer automorphisms which are unipotent polynomially growing in the sense of Bestvina, Feighn and Handel [1], and the maps $f_{k}^{+}$ 
satisfy the conclusions of [1, Theorem 5.1.8]. We do not quote here the statement of this theorem, which would lead us to introduce a lot of technical background, but we freely use in the sequel some consequences of it.

Let $\mathcal{A}$ be a basis of $F_{N}$. We denote by $[g]$ the reduced word, in the basis $\mathcal{A}$, representing the element $g \in F_{N}$. Let $\varphi$ be an automorphism of $F_{N}$. A splitting of $g \in F_{N}$ for $\varphi$ is a way to write $g=g_{1} \cdots g_{n}$ such that:

(i) $n \geqslant 2$,

(ii) for all $i \in\{1, \ldots, n\}, g_{i} \in F_{N} \backslash\{1\}$,

(iii) for all $p \in \mathbb{N}$, for all $i \in\{1, \ldots, n-1\},\left[\varphi^{p}\left(g_{i}\right)\right]\left[\varphi^{p}\left(g_{i+1}\right)\right]=\left[\varphi^{p}\left(g_{i} g_{i+1}\right)\right]$ (this means that no cancellation occurs between $\left[\varphi^{p}\left(g_{i}\right)\right]$ and $\left.\left[\varphi^{p}\left(g_{i+1}\right)\right]\right)$.

In that case, we note $g=g_{1} \cdots g_{n}$, and each $g_{i}$ is called a brick of the splitting.

We now apply that $\left[1\right.$, Theorem 5.1.8] to the given family $\varphi_{k}$ and obtain:

Lemma 5.3 For all $g \in F_{4}$, there exists some $p_{0} \in \mathbb{N}$ such that for all $p \geqslant p_{0}$, $\left[\varphi_{k}^{p}(g)\right]$ and $\left[\varphi_{k}^{-p}(g)\right]$ have a splitting, the bricks of which are either edges or paths of the following labels: $b a^{q} b^{-1}, c a^{q} c^{-1}, b a^{q} c^{-1}$ or $c a^{q} b^{-1}$, for some $q \in \mathbb{Z}$.

Remark 5.4 For the reader who is familiar with the terminology of [1], the edge paths labelled by $b a^{q} b^{-1}, c a^{q} c^{-1}, b a^{q} c^{-1}$ or $c a^{q} b^{-1}$ are precisely the exceptional paths of the improved train-track map $f_{k}$.

Remark 5.5 As a consequence of Lemma 5.3, one can easily check that the sequence $\left(\left|\left[\varphi_{k}^{p}(g)\right]\right|\right)_{p \in \mathbb{N}}$ of lengths of $\left[\varphi_{k}^{p}(g)\right]$ is bounded above by a polynomial of degree 2 in $p$.

It is claimed in Maslakova [17] that there exists a general algorithm to compute the fixed subgroup of a given automorphism of $F_{N}$. There exist some easier algorithms for special cases: for instance, one could use Cohen and Lustig [3] to compute the fixed subgroup of $\varphi_{k}$. In fact, it is sufficient to determines the so called indivisible Nielsen paths; see Bestvina and Handel [2].

Let $\mathcal{N} \mathcal{P}$ denote the set $\left\{a^{q}, b a^{q} b^{-1}, c a^{q} c^{-1} \mid q \in \mathbb{Z}\right\}$. We notice that $\mathcal{N} \mathcal{P} \subseteq \operatorname{Fix}\left(\varphi_{k}\right)$. For an element $g \in F_{4}$, we consider the splitting of $\left[\varphi_{k}^{p}(g)\right]=g_{1} \cdot g_{2} \cdot \ldots \cdot g_{r}$ given by Lemma 5.3. If $g_{i} \in \mathcal{N} \mathcal{P}$ for all $i \in\{1, \ldots, r\}$, then $g \in \operatorname{Fix}\left(\varphi_{k}\right)$. Otherwise, there is some $i_{0}$ such that $g_{i} \in \mathcal{N} \mathcal{P}$ for all $i \in\left\{1, \ldots, i_{0}\right\}$ and $g_{i_{0}+1} \notin \mathcal{N} \mathcal{P}$. For simplicity, we write $\omega_{\varphi_{k}}=\omega_{k}$. Then $\omega_{k}(g)=g_{1} \ldots g_{i_{0}} \omega_{k}\left(g_{i_{0}+1}\right) \in \partial F_{4}$. This shows that $\operatorname{Fix}\left(\varphi_{k}\right)=\left\langle a, b a b^{-1}, c a c^{-1}\right\rangle$. 
Moreover, we thus obtain all the isoglossy classes of limit points of $\varphi_{k}$ by considering all the $\omega_{k}(h)$ with $h \in\left\{b^{ \pm 1}, c^{ \pm 1}, d^{ \pm 1}, b a^{q} c^{-1}, c a^{q} b^{-1} \mid q \in \mathbb{Z}\right\}$. A direct computation gives $\varphi_{k}^{p}(b)=b a^{p}, \varphi_{k}^{p}(c)=c a^{p(k+1)}, \varphi_{k}^{p}\left(b a^{q} c^{-1}\right)=b a^{q-k p} c^{-1}$, $\varphi_{k}^{p}(d)=d c c a^{k+1} c a^{2(k+1)} \cdots c a^{(p-1)(k+1)}, \varphi_{k}\left(d^{-1}\right)=c^{-1} \cdot d^{-1}$. We derive that:

- $\omega_{k}(b)=b a^{+\infty}$,

- $\omega_{k}(c)=\omega_{k}\left(c a^{q} b^{-1}\right)=c a^{+\infty}$,

- $\omega_{k}\left(b^{-1}\right)=\omega_{k}\left(c^{-1}\right)=\omega_{k}\left(d^{-1}\right)=a^{-\infty}$,

- $\omega_{k}\left(b a^{q} c^{-1}\right)=b a^{-\infty}$,

- $\omega_{k}(d)=X_{k}^{+}$,

where $X_{k}^{+}=\omega_{k}(d)=d c c a^{k+1} c a^{2 k+2} c a^{3 k+3} \ldots$ We have thus shown that there are only 5 isoglossy classes in $L_{\varphi_{k}}^{\omega}$, given by $X_{k}^{+}, a^{-\infty}, c a^{+\infty}, b a^{+\infty}, b a^{-\infty}$.

Theorem 5.6 The set $\left\{\varphi_{k} \mid k \in \mathbb{N}\right\}$ is a family of automorphisms of $F_{4}$, such that each $\varphi_{k}$ has a parabolic orbit. The dynamics graph of $\varphi_{k}$ is given in Figure 2. For any $k, k^{\prime}, p, p^{\prime} \in \mathbb{N}, \varphi_{k}^{p}$ and $\varphi_{k^{\prime}}^{p^{\prime}}$ are conjugate if and only if $k=k^{\prime}$ and $p=p^{\prime}$.

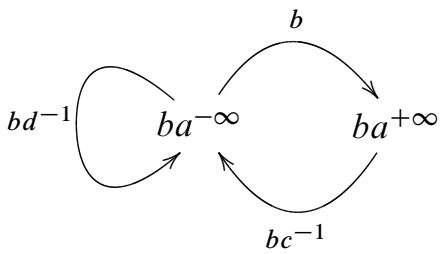

$$
X_{k}^{+}<\frac{d}{<} X_{k}^{-} \quad a^{+\infty} \stackrel{b^{-1}}{\longrightarrow} a^{-\infty} \stackrel{d^{-1}}{\leftarrow} c a^{-\infty} \stackrel{c}{\longrightarrow} c a^{+\infty}
$$

Figure 2. The dynamics graph of $\varphi_{k}$ has 3 connected components. A label $g$ has been added to each edge: it means that $\omega_{k}(g)$ is the endpoint of the edge and $\omega_{k}^{-}(g)$ is the origin of the edge.

Proof We write $\omega_{\varphi_{k}^{-1}}=\omega_{k}^{-}$. Arguing for $\varphi_{k}^{-1}$ as we have done for $\varphi_{k}$, we obtain:

- $\omega_{k}^{-}\left(b^{-1}\right)=\omega_{k}^{-}\left(c^{-1}\right)=a^{+\infty}$,

- $\omega_{k}^{-}(c)=\omega_{k}^{-}\left(d^{-1}\right)=c a^{-\infty}$,

- $\omega_{k}^{-}(b)=b a^{-\infty}$,

- $\omega_{k}^{-}\left(b c^{-1}\right)=b a^{+\infty}$,

- $\omega_{k}^{-}(d)=X_{k}^{-}$,

where $X_{k}^{-}=\omega_{k}^{-}(d)=d a^{k+1} c^{-1} a^{2 k+2} c^{-1} a^{3 k+3} c^{-1} \ldots$ Again, there are only 5 isoglossy classes in $L_{\varphi_{k}^{-1}}^{\omega}$, given by $X_{k}^{-}, a^{+\infty}, c a^{-\infty}, b a^{-\infty}, b a^{+\infty}$. 
Note that $\varphi_{k}\left(b d^{-1}\right)=b a c^{-1} \cdot d^{-1}$ is a splitting for $\varphi_{k}$. Hence $\omega_{k}\left(b d^{-1}\right)=$ $\omega_{k}\left(b a c^{-1}\right)=b a^{-\infty}$. On the other hand, $b \cdot d^{-1}$ is a splitting for $\varphi_{k}^{-1}$. Hence $\omega_{k}^{-}\left(b d^{-1}\right)=\omega_{k}^{-}(b)=b a^{-\infty}$. Thus $b a^{-\infty}$ is parabolic fixed point for $\varphi_{k}$.

Suppose that $\varphi_{k}^{p}$ and $\varphi_{k^{\prime}}^{p^{\prime}}$ are conjugate $\left(k, k^{\prime}, p, p^{\prime} \in \mathbb{N}\right)$ : there exists $\psi \in \operatorname{Aut}\left(F_{4}\right)$ such that $\varphi_{k}^{p}=\psi \varphi_{k^{\prime}}^{p^{\prime}} \psi^{-1}$. Let $M_{k}, M_{k^{\prime}}, P \in \mathrm{GL}(4, \mathbb{Z})$ be the matrices obtained by abelianization of respectively $\varphi_{k}, \varphi_{k^{\prime}}$ and $\psi$. Then

$$
M_{k}^{p}=\left(\begin{array}{cccc}
1 & p & (k+1) p & \frac{1}{2}(k+1) p(p-1) \\
0 & 1 & 0 & 0 \\
0 & 0 & 1 & p \\
0 & 0 & 0 & 1
\end{array}\right) .
$$

Computing $M_{k}^{p} P=P M_{k^{\prime}}^{p^{\prime}}$, one sees that $P$ must have the following shape:

$$
P=\left(\begin{array}{cccc}
\lambda_{1} & \mu_{1} & \mu_{2} & \mu_{3} \\
0 & \lambda_{2} & 0 & \mu_{4} \\
0 & \mu_{5} & \lambda_{3} & \mu_{6} \\
0 & 0 & 0 & \lambda_{4}
\end{array}\right)
$$

with

$$
p^{\prime}\left(k^{\prime}+1\right) \lambda_{3}=p(k+1) \lambda_{1} \quad \text { and } \quad p^{\prime} \lambda_{3}=p \lambda_{4} .
$$

We deduce that $\operatorname{det} P=\lambda_{1} \lambda_{2} \lambda_{3} \lambda_{4}$, and thus $\lambda_{i} \in\{ \pm 1\}$, since $\operatorname{det} P= \pm 1$. From (1) we derive $k=k^{\prime}$ and $p=p^{\prime}$.

\subsection{Parabolic orbits for $N \geqslant 5$}

For any $k \in \mathbb{N}$, consider the automorphism $\alpha_{k}$ of $F_{5}=\langle a, b, c, d, e\rangle$ given by

$$
\begin{aligned}
\alpha_{k}: a & \mapsto a \\
b & \mapsto b a \\
c & \mapsto c a^{k+1} \\
d & \mapsto d c \\
e & \mapsto e .
\end{aligned}
$$

Since the restriction of $\alpha_{k}$ to $\langle a, b, c, d\rangle$ is $\varphi_{k}$, clearly, $\omega_{\alpha_{k}}\left(b d^{-1}\right)=\omega_{\alpha_{k}^{-1}}\left(b a c^{-1}\right)=$ $b a^{-\infty}$ is a parabolic fixed point for $\alpha_{k}$. Considering the abelianization and arguing as previously, we check that if $k \neq k^{\prime}$ and $p \neq p^{\prime}$, then $\alpha_{k}^{p}$ and $\alpha_{k^{\prime}}^{p^{\prime}}$ cannot be conjugate. If $N \geqslant 6$, we split $F_{N}=F_{4} * F_{2} * F_{N-6}$. We first recall some facts about $\operatorname{Out}\left(F_{2}\right)$. It is well known, since Nielsen [18], that the abelianisation morphism from $\operatorname{Out}\left(F_{2}\right)$ 
to $\mathrm{GL}_{2}(\mathbb{Z})$ is an isomorphism. If $M \in \mathrm{SL}_{2}(\mathbb{Z})$ has a trace bigger than 2 , then $M$ has an eigenvalue $\lambda>1$ which is an algebraic unity of a quadratic extension of $\mathbb{Q}$ : we call $\lambda$ the dilatation of $M$. For all $k \in \mathbb{N}$ prime, there exists $M_{k} \in \mathrm{SL}_{2}(\mathbb{Z})$ such that the dilatation $\lambda_{k}$ of $M_{k}$ belongs to $\mathbb{Q}(\sqrt{k}) \backslash \mathbb{Q}$. This implies in particular that for all $p \in \mathbb{N}, \lambda_{k}^{p} \in \mathbb{Q}(\sqrt{k}) \backslash \mathbb{Q}$. We choose $\theta_{k} \in \operatorname{Aut}\left(F_{2}\right)$ in the outer class represented by $M_{k}$. Then the automorphism $\theta_{k}^{p}$ has growth rate equal to $\lambda_{k}^{p}$.

We define $\beta_{k} \in \operatorname{Aut}\left(F_{N}\right)$ by $\beta_{k}=\varphi_{1} * \theta_{k} *$ id, where id is the identity on $F_{N-6}$. Again, $\omega_{\beta_{k}}\left(b d^{-1}\right)=\omega_{\beta_{k}^{-1}}\left(b a c^{-1}\right)=b a^{-\infty}$ is a parabolic fixed point for $\beta_{k}$. Since $\varphi_{1}$ is polynomially growing, it follows that the growth rate of $\beta_{k}^{p}$ is $\lambda_{k}^{p}$ (see for instance [12]). This proves that $\beta_{k}^{p}$ is not conjugate to $\beta_{k^{\prime}}^{p^{\prime}}$ if $k \neq k^{\prime}$ or $p \neq p^{\prime}$, because the growth rate is a conjugacy invariant and because $\mathbb{Q}(\sqrt{k}) \cap \mathbb{Q}\left(\sqrt{k^{\prime}}\right)=\mathbb{Q}$ (if $k$ and $k^{\prime}$ are prime integers).

This finishes the proof Theorem 1.1. In view of Proposition 1.2, it remains to ask the following question, the answer of which we do not know:

Question 5.7 Does there exist an automorphism of $F_{3}$ which has a parabolic orbit?

\section{Dehn twist automorphisms of $F_{2}$}

In this last section, we calculate the dynamics graphs of all the automorphisms in the outer class of $\delta^{n}(n \in \mathbb{Z}, n \neq 0)$, where $\delta$ is the automorphism of $F_{2}=\langle a, b\rangle$ defined by $\delta(a)=a$ and $\delta(b)=b a$.

Let $D \in \operatorname{Out}\left(F_{2}\right)$ be the outer class of $\delta$. As explained in Section 4.2, the automorphisms in the outer class $D^{n}(n \in \mathbb{Z})$ cannot have parabolic orbits. We are going to describe more precisely the dynamics induced on $\partial F_{N}$ by the automorphisms in the outer class $D^{n}(n \in \mathbb{Z}, n \neq 0)$. For that, we pursue the strategy of [6;13], where the interested reader will be able to find details of the following constructions.

The rose $R_{2}$ is the geometric realization of the graph with one vertex and 2 edges. We put an orientation on each edge, and we label them by $a$ and $b$. We can turn $R_{2}$ in a length space by declaring that each edge has length 1 . We represent $D^{n}$ by an homotopy equivalence $f$ of $R_{2}$ defined in the following way: $f$ is the identity on the edge $a$ and linearly sends the edge $b$ to the edge path labelled $b a^{n}$.

The universal cover $\widetilde{R}_{2}$ of $R_{2}$ is a tree, equipped by the action of $F_{2}$ by deck transformations. We lift the labels of the edges of $R_{2}$ to the edges of $\widetilde{R}_{2}$. Equivalently, $\widetilde{R}_{2}$ can be considered as the Cayley graph of $F_{2}$ relative to the generating set $\{a, b\}$. Let $T$ be the tree obtained by contracting in $\widetilde{R}_{2}$ all the edges labelled by $a$ : the action 
of $F_{N}$ on $\widetilde{R}_{2}$ induces an action of $F_{2}$ on $T$ by isometries. We note that the stabilizer of a vertex of $T$ is conjugate to the subgroup $\langle a\rangle \subset F_{N}$ generated by $a$.

As in the geometric case (see Section 4.2) the automorphisms in the outer class $D^{n}$ are in 1:1 correspondence with the lifts of $f$ to $\widetilde{R}_{2}$. Moreover, these lifts of $f$ induce isometries of $T$. More precisely, the isometry $H$ of $T$ associated to the automorphism $\delta^{n} \in D^{n}$ satisfies

$$
\delta^{n}(g) \circ H=H \circ g \quad \forall g \in F_{N},
$$

where the elements of $F_{N}$ are considered as isometries of $T$. Then, for $u \in F_{N}$, the map $H_{u}=u \circ H$ is the isometry of $T$ associated to the automorphism $i_{u} \circ \delta^{n} \in D^{n}$, since $\left(i_{u} \circ \delta^{n}\right)(g) \circ H_{u}=H_{u} \circ g$ holds for all $g \in F_{N}$.

If $H_{u}$ is a hyperbolic isometry of $T$, then $i_{u} \circ \delta^{n}$ has North-South dynamics and the fixed points of $i_{u} \circ \delta^{n}$ are determined by the ends of the axis of $H_{u}$ in $T$; see [13].

If $H_{u}$ is an elliptic isometry, let $P \in T$ be a fixed point of $H_{u}$. There exists some $w \in F_{N}$ such that the stabilizer of $P$ in $F_{N}$ is $w\langle a\rangle w^{-1}$. The fact that $P$ is a fixed point of $H_{u}$ then results in the existence of an integer $k \in \mathbb{Z}$ such that $u \delta^{n}(w)=w a^{k}$. Or equivalently, such that $i_{u} \circ \delta^{n}=i_{w} \circ\left(i_{a^{k}} \circ \delta^{n}\right) \circ i_{w}^{-1}$. Indeed,

$$
\begin{aligned}
i_{u} \circ \delta^{n} & =i_{w a^{k}\left(\delta^{n}(w)\right)^{-1} \circ \delta^{n}} \\
& =i_{w a^{k} \delta^{n}\left(w^{-1}\right)} \circ \delta^{n} \\
& =i_{w} \circ i_{a^{k}} \circ i_{\delta^{n}\left(w^{-1}\right)} \circ \delta^{n} \\
& =i_{w} \circ i_{a^{k}} \circ \delta^{n} \circ i_{w^{-1}} .
\end{aligned}
$$

The dynamics of $\partial\left(i_{u} \circ \delta^{n}\right)$ is thus conjugate to the dynamics of $\partial\left(i_{a^{k}} \circ \delta^{n}\right)$ for some $k \in \mathbb{Z}$. We are now going to study in more detail the automorphisms $i_{a^{k}} \circ \delta^{n}$ for $k \in \mathbb{Z}$, and in particular, to give their dynamics graphs.

The inverse of $i_{a^{k}} \circ \delta^{n}$ is $i_{a^{-k}} \circ \delta^{-n}$. We note that

$$
\begin{aligned}
& i_{a^{k}} \circ \delta^{n}: \quad a \mapsto a \\
& i_{a^{-k}} \circ \delta^{-n}: \quad a \mapsto a \\
& b \mapsto a^{k} b a^{n-k} \\
& b \mapsto a^{-k} b a^{k-n} \\
& b^{-1} \mapsto a^{k-n} b^{-1} a^{-k} \\
& b^{-1} \mapsto a^{n-k} b^{-1} a^{k} .
\end{aligned}
$$

Thus the dynamics of $\partial\left(i_{a_{2}^{k}}^{k} \circ \delta^{n}\right)$ depends on the sign of $k$ and of $n-k$.

Remark 6.1 Let $\sigma \in \operatorname{Aut}\left(F_{N}\right)$ defined by $\sigma(a)=a^{-1}$ and $\sigma(b)=b^{-1}$. We note that $i_{a^{k}} \circ \delta^{n}$ and $i_{a^{n-k}} \circ \delta^{n}$ are conjugate by the involution $\sigma$.

First case Assume $k(n-k)=0$. Since $\delta^{n}$ and $i_{a^{n}} \circ \delta^{n}$ are conjugate by $\sigma$ (see Remark 6.1), we focus on $\delta^{n}$. One can check that $\operatorname{Fix}\left(\delta^{n}\right)=\left\langle a, b a b^{-1}\right\rangle$. Let $X$ be a 
point in $\partial F_{2} \backslash \partial\left\langle a, b a b^{-1}\right\rangle$, and let $x$ be the longest prefix of $X$ in $\left\langle a, b a b^{-1}\right\rangle$. Then $X=x Y$, with no cancellation between $x$ and $Y$, and the first letter of $Y$ is equal to $b$ or to $b^{-1}$. If $Y$ begins by $b$, then $\omega_{\delta^{n}}(Y)=b a^{\infty}$ and $\omega_{\delta^{-n}}(Y)=b a^{-\infty}$. If $Y$ begins by $b^{-1}$, then $\omega_{\delta^{n}}(Y)=a^{-\infty}$ and $\omega_{\delta^{-n}}(Y)=a^{\infty}$. Hence $\delta^{n}$ has 2 isoglossy classes of $\omega$-limit points (with representatives $b a^{\infty}$ and $a^{-\infty}$ ), and $\delta^{-n}$ has 2 isoglossy classes of $\omega$-limit points (with representatives $b a^{-\infty}$ and $a^{\infty}$ ). The dynamics graph of $\delta^{n}$ is given in Figure 3.

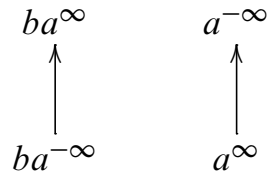

$k=0$

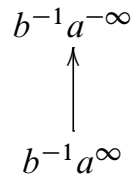

$k=n$

Figure 3. Dynamics graph of $i_{a^{k}} \circ \delta^{n}$ for $k(n-k)=0$

Second case $k(n-k)<0$. We suppose that $k>n$ (from which one deduces the case $k<0$ by using Remark 6.1). The fixed subgroup is $\operatorname{Fix}\left(i_{a^{k}} \circ \delta^{n}\right)=\langle a\rangle$. We note that $\omega_{i_{a} k \circ \delta^{n}}(b)=\omega_{i_{a} k \circ \delta^{n}}\left(b^{-1}\right)=a^{\infty}$ and $\omega_{\left(i_{a} k \circ \delta^{n}\right)^{-1}}(b)=\omega\left(i_{a} k \circ \delta^{n}\right)^{-1}\left(b^{-1}\right)=a^{-\infty}$. It follows that $\partial\left(i_{a^{k}} \circ \delta^{n}\right)$ has North-South dynamics on $\partial F_{2}$; see Figure 4.

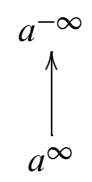

$k<0$

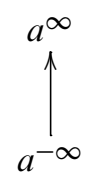

$k>n$

Figure 4. Dynamics graph of $i_{a^{k}} \circ \delta^{n}$ for $k(n-k)<0$

Third case $k(n-k)>0$, ie $0<k<n$. We check that the fixed subgroup is equal to $\operatorname{Fix}\left(i_{a^{k}} \circ \delta^{n}\right)=\langle a\rangle$. We note that $\omega_{i_{a} k \circ \delta^{n}}(b)=a^{\infty}, \omega_{i_{a} k \circ \delta^{n}}\left(b^{-1}\right)=a^{-\infty}$,

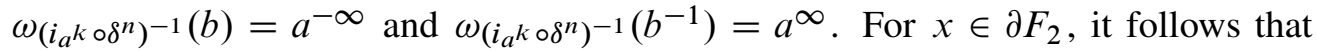

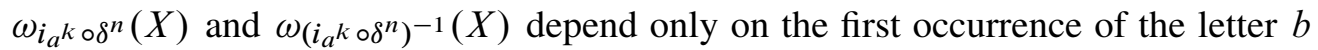

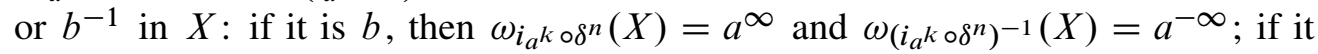

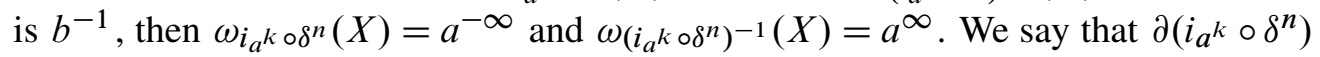
has semi-North-South dynamics on $\partial F_{2}$; see Figure 5. 


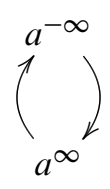

Figure 5. Dynamics graph of $i_{a} k \circ \delta^{n}$ for $k(n-k)>0$

\section{References}

[1] M Bestvina, M Feighn, M Handel, The Tits alternative for $\operatorname{Out}\left(F_{n}\right)$ I: Dynamics of exponentially-growing automorphisms, Ann. of Math. 151 (2000) 517-623 MR1765705

[2] M Bestvina, M Handel, Train tracks and automorphisms of free groups, Ann. of Math. 135 (1992) 1-51 MR1147956

[3] MM Cohen, M Lustig, On the dynamics and the fixed subgroup of a free group automorphism, Invent. Math. 96 (1989) 613-638 MR996557

[4] D Cooper, Automorphisms of free groups have finitely generated fixed point sets, J. Algebra 111 (1987) 453-456 MR916179

[5] M Feighn, M Handel, The recognition theorem for $\operatorname{Out}\left(F_{n}\right)$, Groups Geom. Dyn. 5 (2011) 39-106 MR2763779

[6] D Gaboriau, A Jaeger, G Levitt, M Lustig, An index for counting fixed points of automorphisms of free groups, Duke Math. J. 93 (1998) 425-452 MR1626723

[7] É Ghys, P de la Harpe, editors, Sur les groupes hyperboliques d'après Mikhael Gromov, Progress in Math. 83, Birkhäuser, Boston (1990) MR1086648 Papers from the Swiss Seminar on Hyperbolic Groups held in Bern, 1988

[8] M Handel, L Mosher, Subgroup classification in $\operatorname{Out}\left(F_{n}\right)$ arXiv:0908.1255

[9] M Handel, W P Thurston, New proofs of some results of Nielsen, Adv. in Math. 56 (1985) 173-191 MR788938

[10] A Hilion, Dynamique des automorphismes des groupes libres, PhD thesis, Université Toulouse 3 (2004)

[11] G Levitt, Homéomorphismes dynamiquement simples de l'ensemble de Cantor, Enseign. Math. 44 (1998) 279-289 MR1659216

[12] G Levitt, Counting growth types of automorphisms of free groups, Geom. Funct. Anal. 19 (2009) 1119-1146 MR2570318

[13] G Levitt, M Lustig, Most automorphisms of a hyperbolic group have very simple dynamics, Ann. Sci. École Norm. Sup. 33 (2000) 507-517 MR1832822

[14] G Levitt, M Lustig, Periodic ends, growth rates, Hölder dynamics for automorphisms of free groups, Comment. Math. Helv. 75 (2000) 415-429 MR1793796 
[15] G Levitt, M Lustig, Irreducible automorphisms of $F_{n}$ have north-south dynamics on compactified outer space, J. Inst. Math. Jussieu 2 (2003) 59-72 MR1955207

[16] G Levitt, M Lustig, Automorphisms of free groups have asymptotically periodic dynamics, J. Reine Angew. Math. 619 (2008) 1-36 MR2414945

[17] OS Maslakova, The fixed point group of a free group automorphism, Algebra Logika 42 (2003) 422-472, 510-511 MR2017513

[18] J Nielsen, Die Isomorphismen der allgemeinen, unendlichen Gruppe mit zwei Erzeugenden, Math. Ann. 78 (1917) 385-397 MR1511907

[19] J Nielsen, Untersuchungen zur Topologie der geschlossenen zweiseitigen Flächen, Acta Math. 50 (1927) 189-358 MR1555256

[20] J Nielsen, Untersuchungen zur Topologie der geschlossenen zweiseitigen Flächen. II, Acta Math. 53 (1929) 1-76 MR1555290

[21] J Nielsen, Untersuchungen zur Topologie der geschlossenen zweiseitigen Flächen. III, Acta Math. 58 (1932) 87-167 MR1555345

Mathematiques, LATP - UMR 7353 Aix-Marseille Université

Avenue de l'escadrille Normandie-Niémen, 13397 Marseille Cedex 20, France

arnaud.hilion@univ-amu.fr

http://junon.u-3mrs.fr/hilion/

Received: 22 September 2011 Revised: 28 January 2012 